GLASNIK MATEMATIČKI

Vol. 38(58)(2003), $29-44$

\title{
ON A GENERALIZATION OF THE SINE FUNCTION
}

\author{
Tomasz Szostok \\ Silesian University, Poland
}

\begin{abstract}
A function $s(x, y)=\inf _{\lambda \in \mathbb{R}} \frac{\|x+\lambda y\|}{\|x\|}$ strictly connected with Birkhoff-James orthogonality is considered. This function may be used in functional equations theory, to provide unconditional equations in place of orthogonal equations in the sense of Birkhoff-James. Moreover, we deal with another generalization of the sine function which, in particular leads to a characterization of inner product spaces.
\end{abstract}

\section{Introduction}

The main idea of the present paper comes from the theory of conditional functional equations. More precisely we talk about the so called orthogonal equations. Consider, for example, the well known Cauchy equation

$$
f(x+y)=f(x)+f(y) .
$$

Any solution of this equation is called an additive function. Let us now consider the related conditional equation

$$
x \perp y \Rightarrow f(x+y)=f(x)+f(y) .
$$

The condition $x \perp y$ may be understood in various ways. For instance, if we deal with functions defined on inner product spaces then we can use the orthogonality defined by an inner product. In an abstract normed linear space the orthogonality has to be defined in another way. The most widely used kinds of orthogonalities are:

James orthogonality

$$
x \perp_{J} y \Leftrightarrow\|x+y\|=\|x-y\| ;
$$

2000 Mathematics Subject Classification. 39B52, 46B20.

Key words and phrases. Birkhoff-James orthogonality, functional equation, inner product space. 
Birkhoff-James orthogonality

$$
x \perp_{B J} y \Leftrightarrow(\|x+\lambda y\| \geq\|x\| \quad \text { for all } \lambda \in \mathbb{R}) ;
$$

and Pythagorean orthogonality

$$
x \perp_{P} y \Leftrightarrow\|x+y\|^{2}=\|x\|^{2}+\|y\|^{2} .
$$

Clearly, every solution of equation (1.1) is a solution of (1.2). The converse implication is false, in general. For example if $X$ is an inner product space and $f: X \rightarrow \mathbb{R}$ is defined by the formula $f(x):=\|x\|^{2}$, then $f$ is a solution of (1.2) that is not additive. Have a look at the equation

$$
f(x+y)=g\left(\frac{\|x-y\|}{\|x+y\|}\right)[f(x)+f(y)] .
$$

It is an unconditional equation and it may be assumed for almost all values of $x$ and $y$. Every additive function $f$ is a solution of this equation ( with function $g=1$ ) and $f(x)=\|x\|^{2}$ is also a solution of this equation ( with $\left.g(a)=\frac{2}{1+a^{2}}\right)$. That means that equation (1.3) preserves the most important solutions of (1.2). Further taking here $x, y \neq 0, x \perp_{J} y$ we obtain

$$
x, y \neq 0, x \perp_{J} y \Rightarrow f(x+y)=g(1)[f(x)+f(y)],
$$

which means that we get a modified version of James orthogonal additivity as a special case of equation (1.3). Consequently, this equation can be easily solved with help of results concerning orthogonally additive functions (see [2]). In the case of normed spaces which are not inner product spaces Gy. Szabó papers devoted to James orthogonal additivity [3] and [4] are useful. Such problems were considered in papers paper [5] and [6]. A natural question arises whether a similar procedure may be applied with respect to another orthogonalities. In order to deal with the Birkhoff-James orthogonality we may consider a function similar to the quotient $\frac{\|x-y\|}{\|x+y\|}$ connected with the James orthogonality, namely

$$
s(x, y):=\inf _{\lambda \in \mathbb{R}} \frac{\|x+\lambda y\|}{\|x\|} .
$$

Then the following equation is to be considered

$$
f(x+y)=g(s(x, y))[f(x)+f(y)] .
$$

However, our present goal is only to examine some properties of the function $s$ just introduced.

\section{Results}

We begin with the following 
Definition 2.1. Let $(X,\|\cdot\|)$ be a real normed linear space. Define a function $s: X \times X \rightarrow \mathbb{R}$ by the following formula:

$$
s(x, y):= \begin{cases}\inf _{\lambda \in \mathbb{R}} \frac{\|x+\lambda y\|}{\|x\|}, & x \neq 0 \\ 1, & x=0 .\end{cases}
$$

Note that, actually, the infimum in definition of $s$ is achieved and consequently may be replaced by the minimum. The following properties of function $s$ are obvious.

Proposition 2.2. Let $(X,\|\cdot\|)$ be a real normed linear space and let $\alpha, \beta$ be some nonzero real numbers. Then $s(\alpha x, \beta y)=s(x, y)$ for all $x, y \in X$.

Further function s takes all its values in the interval $[0,1]$, and $s(x, y)=0$ if and only if $x, y$ are linearly dependent.

Function $s$ is strictly connected with the Birkhoff-James orthogonality. Namely we have the following simple

REMARK 2.3. Let $x, y$ be given elements of a normed linear space $X(\|\cdot\|)$. Then

$$
x \perp_{B J} y \Leftrightarrow s(x, y)=1 .
$$

Remark 2.4. Let $(X,(\cdot \mid \cdot))$ be an inner product space. Then

$$
s(x, y)=\sqrt{1-\frac{(x \mid y)^{2}}{\|x\|^{2}\|y\|^{2}}} \text { for all } x, y \in X \backslash\{0\} ;
$$

in particular $s(x, y)=s(y, x)$. On the other hand if $(X,\|\cdot\|)$ is a normed space of dimension at least 3 and we have $s(x, y)=s(y, x)$ for all $x, y \in X$, then $X$ has to be an inner product space.

Proof. Fix $x, y \in X x, y \neq 0$ and put $a:=\|x\|^{2}, b:=\|y\|^{2}, c:=(x \mid y)$. We shall determine the real number $\lambda_{0}$ such that for this number the expression $h(\lambda):=\frac{\|x+\lambda y\|}{\|x\|}$ is minimal. Since $h$ is nonnegative it suffices to consider its square:

$$
\frac{\|x+\lambda y\|^{2}}{\|x\|^{2}}=\frac{a+2 \lambda c+\lambda^{2} b}{a} .
$$

Obviously, the latter expression (and hence also $h$ ) is minimal for $\lambda_{0}=-\frac{c}{b}$. Using this value of $\lambda$ we get

$$
\begin{gathered}
s(x, y)=\sqrt{\frac{a+2 \lambda_{0} c+\lambda_{0}^{2} b}{a}}=\sqrt{\frac{a-2 \frac{c}{b} c+\frac{c^{2}}{b^{2}} b}{a}} \\
=\sqrt{1-\frac{\frac{c^{2}}{b}}{a}}=\sqrt{1-\frac{c^{2}}{a b}}=\sqrt{1-\frac{(x \mid y)^{2}}{\|x\|^{2}\|y\|^{2}}} .
\end{gathered}
$$

Now, assume that $X$ is a normed space of dimension at least 3 , such that we have $s(x, y)=s(y, x)$ for all $x, y \in X$. From Remark 2.3 we infer that 
$x \perp_{B J} y \Leftrightarrow y \perp_{B J} x$. The symmetry of the Birkhoff-James orthogonality in a space with dimension greater than 2 forces this space to be an inner product space (see [1]).

What about continuity of function $s$ ? If we interpret $s$ as the absolute value of the sinus of the angle between the vectors $x$ and $y$, then we cannot expect the continuity of $s$ at a point $(x, y)$ such that one of the vectors $x, y$ is equal to zero. However, except for these points, the function $s$ is continuous.

THEOREM 2.5. Let $(X,\|\cdot\|)$ be a real normed linear space of dimension at least 2. Then the function

$$
s_{\mid(X \backslash\{0\}) \times(X \backslash\{0\})}
$$

is continuous.

Proof. Since $s(X \times X) \subset[0,1]$, we infer that

$$
\lim \sup _{n \rightarrow \infty} s\left(x_{n}, y_{n}\right)
$$

is finite. Now, take $(x, y) \in X \times X, \quad x, y \neq 0$ and assume that there exist sequences $\left(x_{n}, y_{n}\right) \rightarrow(x, y) \leftarrow\left(x_{n}^{1}, y_{n}^{1}\right)$ such that

$$
s\left(x_{n}, y_{n}\right) \rightarrow \alpha \text { and } s\left(x_{n}^{1}, y_{n}^{1}\right) \rightarrow \alpha_{1}
$$

for some real numbers $\alpha \neq \alpha_{1}$. Assume also that $x_{n}, y_{n}, x_{n}^{1}, y_{n}^{1} \neq 0$. We are going to show that this assumption leads to a contradiction. Assume that $\alpha>\alpha_{1}$ and denote $\varepsilon:=\alpha-\alpha_{1}$. Since $x \neq 0$, we can replace $(x, y)$ by $\left(\frac{x}{\|x\|}, y\right)$ and do the same with the coordinates of $\left(x_{n}, y_{n}\right)$ and $\left(x_{n}^{1}, y_{n}^{1}\right)$. The resulting points will still have all the properties assumed. Consequently there is no loss of generality in assuming that $\|x\|=\left\|x_{n}\right\|=\left\|x_{n}^{1}\right\|=1$ for all $n \in \mathbb{N}$ whence

$$
\min _{\lambda}\left\|x_{n}+\lambda y_{n}\right\| \rightarrow \alpha \text { and } \min _{\lambda}\left\|x_{n}^{1}+\lambda y_{n}^{1}\right\| \rightarrow \alpha_{1}
$$

Thus

$$
\left\|x_{n}+\lambda_{n} y_{n}\right\| \rightarrow \alpha \text { and }\left\|x_{n}^{1}+\lambda_{n}^{1} y_{n}^{1}\right\| \rightarrow \alpha_{1}
$$

where $\lambda_{n}$ and $\lambda_{n}^{1}$ are such real numbers that the considered expressions are minimal; moreover,

$$
\left\|x_{n}+\lambda_{n} y_{n}\right\| \leq\left\|x_{n}+\lambda y_{n}\right\| \quad \text { for all } n \in \mathbb{N} \text { and all } \lambda \in \mathbb{R} .
$$

We have also $\left\|x_{n}^{1}+\lambda_{n}^{1} y_{n}^{1}\right\|=s\left(x_{n}^{1}, y_{n}^{1}\right) \leq 1, n \in \mathbb{N}$. On the other hand

$$
\left\|x_{n}^{1}+\lambda_{n}^{1} y_{n}^{1}\right\| \geq\left|\lambda_{n}^{1}\right|\left\|y_{n}^{1}\right\|-\left\|x_{n}^{1}\right\|, \quad n \in \mathbb{N} .
$$

These conditions together with $y_{n}^{1} \rightarrow y \neq 0$ mean that the sequence $\left(\lambda_{n}^{1}\right)$ is bounded. Consequently, without loss of generality, we may assume that there is a real number $\lambda_{1}$ such that $\lambda_{n}^{1} \rightarrow \lambda_{1}$.

Note that

$$
\left|\left\|x_{n}+\lambda_{1} y_{n}\right\|-\left\|x_{n}^{1}+\lambda_{1} y_{n}^{1}\right\|\right|<\frac{\varepsilon}{9},
$$


as well as

$$
\left|\left\|x_{n}^{1}+\lambda_{1} y_{n}^{1}\right\|-\left\|x_{n}^{1}+\lambda_{n}^{1} y_{n}^{1}\right\|\right|<\frac{\varepsilon}{9}
$$

$$
\left|\alpha_{1}-\left\|x_{n}^{1}+\lambda_{n}^{1} y_{n}^{1}\right\|\right|<\frac{\varepsilon}{9}
$$

for almost all $n \in \mathbb{N}$ and, therefore,

$$
\left|\alpha_{1}-\left\|x_{n}+\lambda_{1} y_{n}\right\|\right|<\frac{\varepsilon}{9}+\frac{\varepsilon}{9}+\frac{\varepsilon}{9}=\frac{\varepsilon}{3} .
$$

for almost all $n \in \mathbb{N}$ say $n \geq n_{0}$. On the other hand

$$
\left\|x_{n}+\lambda_{n} y_{n}\right\|>\alpha-\frac{\varepsilon}{3}
$$

say for $n \geq n_{1}$. Finally, using (2.3), and (2.4), we conclude that for all $n \geq$ $\max \left(n_{0}, n_{1}\right)$ one has

$$
\left\|x_{n}+\lambda_{n} y_{n}\right\|>\alpha-\frac{\varepsilon}{3}>\alpha_{1}+\frac{\varepsilon}{3}>\left\|x_{n}+\lambda_{1} y_{n}\right\|,
$$

which contradicts (2.2). This contradiction shows that for every pair $(x, y) \in$ $X \times X, \quad x, y \neq 0$ the function $s$ has a limit at this point. To finish the proof it is enough to observe that we can take $\left(x_{n}, y_{n}\right)$ equal to $(x, y)$ in the first part of the proof.

As we already mentioned the function $s$ can be viewed as the absolute value of a sinus of the angle between the vectors $x$ and $y$. Several further facts about function $s$ can also be observed. Especially, in inner product spaces this function has many interesting properties. For example, our next remark states that in an inner product space in every isosceles triangle the absolute values of the sinus of the angle between the median and the both equal sides are the same.

REMARK 2.6. If $(X,(\cdot \mid \cdot))$ is a real inner product space, then for all $x, y \in$ $X$, with $\|x\|=\|y\|$ one has

$$
s(x, x+y)=s(y, x+y) .
$$

With the aid of function $s$ we are also able to define a real function which stands for an anologue of sinus in a given normed space. To do this we need earlier the following

REMARK 2.7. Let $(X,\|\cdot\|)$ be a normed linear space and let $x, y \in X$. If $a, b, c, d$ are such real numbers that $\angle((1,0),(a, b))=\angle((1,0),(c, d))$, then $s(x, a x+b y)=s(x, c x+d y)$.

This is a direct consequence of Proposition 2.2. Now we are able to formulate the following definition. 
Definition 2.8. Let $(X,\|\cdot\|)$ be a real normed linear space of dimension at least 2. Take $x, y \in X$, with $\|x\|=\|y\|=1$ and such that $x \perp_{B J} y$. Define $a$ function $\phi_{x, y}: \mathbb{R} \rightarrow \mathbb{R}$ by the following formula

$$
\phi_{x, y}(t):=\operatorname{sgn} b \cdot s(x, a x+b y), \quad t \in \mathbb{R},
$$

where $a, b \in \mathbb{R}$ are such that the angle between the vector $(a, b)$ and the vector $(1,0)$ is equal to $t$.

REMARK 2.9. If $(X,(\cdot \mid \cdot))$ is a real inner product space and $t \in\left(0, \frac{\pi}{2}\right)$, then for all $x, y \in X$, with $x \perp y,\|x\|=\|y\|=1$ we have

$$
\phi_{x, y}\left(\frac{\pi}{2}+t\right)=\phi_{x, y}\left(\frac{\pi}{2}-t\right) .
$$

Proof. Fix $x, y \in X$ satisfying the assumptions of the theorem and put $z:=a x+b y$ where $a, b$ are any real numbers satisfying the equality $\frac{b}{a}=\tan \left(\frac{\pi}{2}+t\right)$. Then

$$
\begin{aligned}
\phi_{x, y}\left(\frac{\pi}{2}+t\right) & =s(x, z)=s(z, x)=s(-z, x) \\
& =s(-a x-b y, x)=s(-a x-b y,-2 a x) .
\end{aligned}
$$

Now, using Remark 2.6, we get

$$
s(-a x-b y,-2 a x)=s(-a x+b y,-2 a x)
$$

and further

$$
s(-a x+b y,-2 a x)=s(-a x+b y, x)=s(x,-a x+b y)=\phi_{x, y}\left(\frac{\pi}{2}-t\right) .
$$

Thus the remark has been proved.

EXAMPLE 2.10. If $(X,(\cdot \mid \cdot))$ is a real inner product space, then for all unit vectors $x, y \in X$ such that $x \perp y$ and for all $t \in \mathbb{R}$ we have $\phi_{x, y}(t)=\sin t$.

Proof. Take $x \in X,\|x\|=1$ and $y \in X$ such that $y \perp x,\|y\|=1$. Then for every $z$ of the form $z=a x+b y$ we have $\|z\|=\sqrt{a^{2}+b^{2}}$. Take a $t \in\left(0, \frac{\pi}{2}\right)$ and write

$$
\begin{gathered}
\phi_{x, y}(t)=s(x, x+\tan t y)=\min _{\lambda \in \mathbb{R}} \frac{\|x+\lambda(x+\tan t y)\|}{\|x\|} \\
=\sqrt{\min _{\lambda \in \mathbb{R}}\left((1+\lambda)^{2}+\lambda^{2} \tan ^{2} t\right)} .
\end{gathered}
$$

Obviously the above formula as a function of $\lambda$ attains its minimum at $\lambda=$ $-\cos ^{2} t$ whence

$$
\phi_{x, y}(t)=\sqrt{\left(1-\cos ^{2} t\right)^{2}+\cos ^{4} t \frac{\sin ^{2} t}{\cos ^{2} t}}=\sin t .
$$

If now $t \in\left(\frac{\pi}{2}, \pi\right)$ then the desired equality results from Remark 2.9. For $t \in(\pi, 2 \pi)$ we also have $\phi_{x, y}(t)=\sin t$ because $s\left(x_{1},-x_{2}\right)=s\left(x_{1}, x_{2}\right)$. For 
bigger values of $t$ we only need to note that $\phi_{x, y}$ is periodic with period equal to $2 \pi$. For $t=\pi$ we have $\phi_{x, y}(t)=0$, if $t=\frac{\pi}{2}$ then we have $\phi_{x, y}(t)=1$.

It is easy to check that in normed spaces which are not inner product spaces functions $\phi_{x, y}$ fail to be the sine function, in general. Have a look at the following example.

EXAmple 2.11. Consider space $\mathbb{R}^{2}$ with the norm $\|(a, b)\|=\max \{|a|,|b|\}$. Then

$$
\phi_{(1,0),(0,1)}(t)= \begin{cases}\frac{\tan t}{1+\tan t}, & t \in\left[0, \frac{\pi}{2}\right), \\ 1, & t=\frac{\pi}{2} .\end{cases}
$$

Natural is the question whether the converse of Example 2.10 holds true. The answer is positive and we shall give it later on in this paper. Namely, among others it will be proved that if in a 2-dimensional space one can find vectors $x, y,\|x\|=\|y\|=1, x \perp_{B J} y$ such that $\phi_{x, y}=\sin$, then this space must be an inner product one. However we have to start with the following lemma.

LEMMA 2.12. Let $f, g:(a, b) \rightarrow \mathbb{R}$ with $-\infty<a<b<\infty$ be given concave functions. If there exists a point $x_{0} \in(a, b)$ such that $g\left(x_{0}\right)>f\left(x_{0}\right)$, then there exists a straight line $l:=\{(x, c x+d): x \in \mathbb{R}\}$ which is not horizontal, has a common point with the graph of $g$ and satisfies the following condition: $c x+d>f(x)+\varepsilon$ for all $x \in(a, b)$ and some $\varepsilon>0$.

Proof. Put $\varepsilon:=\frac{g\left(x_{0}\right)-f\left(x_{0}\right)}{2}$ and let us distinguish two cases:

$1^{0}$ either $f_{+}^{\prime} \neq 0$ or $f_{-}^{\prime} \neq 0$.

$2^{0} f^{\prime}\left(x_{0}\right)=0$.

If $1^{0}$ occurs, then there exists a nontrivial straight line $\tilde{l}=\{(x, \alpha x+\tilde{\beta})$ : $x \in \mathbb{R}\}$ with $\alpha \neq 0$ that supports the graph of $f$ at the point $\left(x_{0}, f\left(x_{0}\right)\right)$ and it sufficies to take $l:=\{(x, \alpha x+\beta): x \in \mathbb{R}\}$ where $\beta:=\tilde{\beta}+2 \varepsilon$.

In the case $2^{0}$ we have $f^{\prime}\left(x_{0}\right)=0$. Then function $f$ has a local maximum at $x_{0}$, further from the concavity of this function we infer that

$$
f(x) \leq f\left(x_{0}\right)<g\left(x_{0}\right)
$$

for all $x \in(a, b)$. Define $c:=\frac{\varepsilon}{x_{0}-a}$ and $d:=g\left(x_{0}\right)-\frac{\varepsilon x_{0}}{x_{0}-a}$. Then the function $h(x)=c x+d$ is increasing and

$$
h(a)=c a+d=g\left(x_{0}\right)-\varepsilon>\max _{x \in(a, b)} f(x)+\varepsilon,
$$

which means that the line defined by the formula $y=c x+d$ satisfies the latter of the conditions desired. To finish the proof it is enough to note that

$$
c x_{0}+d=g\left(x_{0}\right)
$$

i.e. this line has a common point with the graph of $g$. 
LEMma 2.13. Let $(X,\|\cdot\|)$ be a normed space. If $x, y \in X$ are unit vectors, then the set

$$
S:=\left\{(a, b) \in \mathbb{R}^{2}:\|a x+b y\|=1,|a|<1, b>0\right\}
$$

yields a graph of some concave function $f:(-1,1) \rightarrow(0, \infty)$.

Proof. For the sake of brevity we shall be writing $\|(a, b)\|$ instead of $\|a x+b y\|$. Note that for every $t \in(-1,1)$ there exists $f(t)>0$ such that the point $(t, f(t))$ is an element of $S$. Indeed, $\|(t, 0)\|=|t|<1$ and, on the other hand, for every $t \in(-1,1)$ one has

$$
\lim _{s \rightarrow \infty}\|(t, s)\|=\infty
$$

Now we are going to show that for every $t \in(-1,1)$ there exists exactly one corresponding point $f(t)$. Suppose that

$$
\left\|\left(t, s_{1}\right)\right\|=\left\|\left(t, s_{2}\right)\right\|=1
$$

for some real numbers $s_{1}, s_{2}$ and some $t \in(-1,1)$. Assume that $s_{1} \geq s_{2}$, then $\left(t, s_{2}\right)=\lambda\left(t, s_{1}\right)+(1-\lambda)(t, 0)$ and $s_{2}=\lambda s_{1}$ for some $\lambda \in[0,1]$. We shall show that $\lambda=1$. Indeed, consider the case of $t>0$ and define

$$
u:=\alpha\left(t, s_{1}\right)+(1-\alpha)(1,0)
$$

for $\alpha=\frac{\lambda}{t+\lambda-\lambda t}$. Directly from the definition of $u$ we infer that $\|u\| \leq 1$. Put $\beta:=\frac{1}{t+\lambda-\lambda t}$, then $u=\beta\left(t, s_{2}\right)$ whence $1 \geq\|u\|=\beta \geq 1$, i.e. $\beta=1$, which implies that $\lambda=1$, and consequently, $s_{1}=s_{2}$. If we had $t<0$, it would be sufficient to repeat the above calculations taking

$$
u:=\alpha\left(t, s_{1}\right)+(1-\alpha)(-1,0)
$$

with $\alpha=\frac{\lambda}{\lambda t+\lambda-t}$ and $\beta:=\frac{1}{\lambda t+\lambda-t}$. For $t=0$ the equality results directly from (2.8).

Till now we have shown that there exists a function $f$ such that the set $S$ is a graph of this function. To finish the proof we have to show that this function is concave. To this end take $t_{1}, t_{2} \in(-1,1)$ and $\alpha \in(0,1)$. Then

$$
\left\|\alpha\left(t_{1}, f\left(t_{1}\right)\right)+(1-\alpha)\left(t_{2}, f\left(t_{2}\right)\right)\right\| \leq 1
$$

on the other hand from the definition of $f$ we have

$$
\left\|\left(\alpha t_{1}+(1-\alpha) t_{2}, f\left(\alpha t_{1}+(1-\alpha) t_{2}\right)\right)\right\|=1 .
$$

Further, equation (2.9) implies the existence of exactly one $s \geq \alpha f\left(t_{1}\right)+(1-$ $\alpha) f\left(t_{2}\right)$ such that $\left\|\alpha t_{1}+(1-\alpha) t_{2}, s\right\|=1$. Therefore, from equation $(2.10)$ we infer that $s=f\left(\alpha t_{1}+(1-\alpha) t_{2}\right)$, which shows the concavity of $f$.

REMARK 2.14. Let $(X,\|\cdot\|)$ be a real normed linear space. Take $x, y \in X$ such that, $\|x\|=\|y\|=1$ and $x \perp_{B J} y$. If $a, b \in \mathbb{R}$ are such that $\|a x+b y\|=\alpha$, then $|a| \leq \alpha$. 
Remark 2.15. Let $(X,\|\cdot\|)$ be a real normed linear space. Take $x, y \in X$ such that, $\|x\|=\|y\|=1$ and $x \perp_{B J} y$. Let $f:(-1,1) \rightarrow \mathbb{R}$ be such a function that

$$
\left\{(a, b) \in \mathbb{R}^{2}:\|a x+b y\|=1,|a|<1, b>0\right\}=\operatorname{Gr} f .
$$

Let further $\alpha$ be a given positive number. Define the function $\psi_{\alpha}:(1-\alpha, 1+$ $\alpha) \rightarrow \mathbb{R}$ by the formula $\psi_{\alpha}(a):=\alpha f\left(\frac{1}{\alpha}(a-1)\right)$. Then the set

$$
S_{\alpha}:=\left\{(a, b) \in \mathbb{R}^{2}:\|x-(a x+b y)\|=\alpha,|a-1|<\alpha, b>0\right\} .
$$

coincides with the graph of $\psi_{\alpha}$. Moreover the (finite) limits of $\psi_{\alpha}$ at the endpoints of the domain exist and the following equalities hold:

$$
\begin{aligned}
& \lim _{a \rightarrow 1-\alpha} \psi_{\alpha}(a)=\sup \{b:\|(1-\alpha, b)\|=\alpha\}, \\
& \lim _{a \rightarrow 1+\alpha} \psi_{\alpha}(a)=\sup \{b:\|(1+\alpha, b)\|=\alpha\} .
\end{aligned}
$$

Proof. We shall present only a sketch of the proof. One has to show that for every $(a, b) \in S_{\alpha}$ we get $b=\psi_{\alpha}(a)$ (the converse implication can be proved similarly). From the definition of $S_{\alpha}$ we have

$$
\|(a x+b y)-x\|=\alpha,
$$

whence

$$
\left\|\frac{1}{\alpha}((a-1) x+b y)\right\|=1 .
$$

We have also $|a-1|<\alpha$ i.e. $\left|\frac{1}{\alpha}(a-1)\right|<1$ whence, $\frac{1}{\alpha} b=f\left(\frac{1}{\alpha}(a-1)\right)$ and, consequently, $b=\psi_{\alpha}(a)$.

The second statement results from the following facts: every concave function defined on an interval is monotone in some neighbourhoods of the ends of this interval, and the 2-dimensional unit sphere is a compact set.

Lemma 2.16. Let $(X,\|\cdot\|)$ be a real normed linear space. Take $x, y \in X$, such that $\|x\|=\|y\|=1$ and $x \perp_{B J} y$. Let $f_{0}:(-1,1) \rightarrow \mathbb{R}$ such a function that

$$
\operatorname{Gr} f_{0}=\left\{(a, b) \in \mathbb{R}^{2}:\|a x+b y\|=1,|a|<1, b>0\right\} .
$$

Take also $t \in\left(0, \frac{\pi}{2}\right)$ and put $l:=\{(a,(\tan t) a): a \in \mathbb{R}\}$. Extend $f_{0}$ to $a$ continuous function, $f:[-1,1] \rightarrow \mathbb{R}$. Let further $\psi_{\alpha}$ be defined in the same way as in Remark 2.15. Then $\phi_{x, y}(t)=\alpha_{0}$ if and only if

$$
l \cap \operatorname{Gr} \psi_{\alpha_{0}} \neq \emptyset \text { and } l \cap \operatorname{Gr} \psi_{\alpha}=\emptyset \quad \text { for every } \alpha \in\left(0, \alpha_{0}\right)
$$

( in the case of $t \in\left(\frac{\pi}{2}, \pi\right)$ we would have to define and use an anologue of function $\psi_{\alpha}$ with $(a-1)$ replaced by $\left.(a+1)\right)$.

Proof. If $\phi_{x, y}(t)=\alpha_{0}$, then

$$
\min _{\lambda \in \mathbb{R}}\|x+\lambda(x+\tan t y)\|=\alpha_{0} .
$$


Now we can find a $\lambda_{0} \in \mathbb{R}$ such that the above minimum is achieved for $\lambda$ equal to $-\lambda_{0}$. Then we have $\left\|x-\lambda_{0}(x+\tan t y)\right\|=\alpha_{0}$. Since $\alpha_{0} \leq 1$, we have $\left\|\left(1-\lambda_{0}\right) x-\lambda_{0} \tan t y\right\| \leq 1$. From Remark 2.14 we infer that $\lambda_{0} \geq 0$ and, moreover, $\lambda_{0}(x+\tan t y) \in \partial \mathrm{K}\left(x, \alpha_{0}\right)$, where $\partial \mathrm{K}\left(x, \alpha_{0}\right)$ stands for a sphere centered at $x$ and having radius equal to $\alpha_{0}$. Let us write these facts in the following way

$$
\lambda_{0}(x+\tan t y) \in \partial \mathrm{K}^{+}\left(x, \alpha_{0}\right):=\left\{a x+b y:\|x-(a x+b y)\|=\alpha_{0}, b \geq 0\right\} .
$$

On the other hand equation (2.11) implies that

$$
\lambda(x+\tan t y) \notin \operatorname{Int} \mathrm{K}\left(x, \alpha_{0}\right) .
$$

for all $\lambda \in \mathbb{R}$. Have a closer look at the set $\partial \mathrm{K}^{+}\left((1,0), \alpha_{0}\right)$ (the point $((1,0)$ is here being identified with the vector $x$ ). From Remark 2.14 it follows that this set can be written in the following way.

$$
\partial \mathrm{K}^{+}\left((1,0), \alpha_{0}\right)=\left\{(a, b) \in \mathbb{R}^{2}:\|(1,0)-(a, b)\|=\alpha_{0}, b \geq 0\right\}=K_{1} \cup K_{2}
$$

where

$$
K_{1}:=\left\{(a, b) \in \mathbb{R}^{2}:\|(1,0)-(a, b)\|=\alpha_{0}, a \in(1-\alpha, 1+\alpha), b \geq 0\right\}
$$

and

$$
\left.K_{2}:=\left\{(a, b) \in \mathbb{R}^{2}:\|(1,0)-(a, b)\|=\alpha_{0}, a \in\{1-\alpha, 1+\alpha\}\right), b \geq 0\right\} .
$$

Let us consider the case when $\left(\lambda_{0}, \lambda_{0} \tan t\right) \in K_{1}$. We have then

$$
K_{1} \subset \operatorname{Gr} \psi_{\alpha_{0}}
$$

whence $l \cap \operatorname{Gr} \psi_{\alpha_{0}} \neq \emptyset$, which means that the first assertion is true. Moreover, from condition (2.12) we infer that $l$ not only has a common point with the graph of considered function but also

$$
\|(1,0)-(\lambda, \lambda \tan t)\| \geq \alpha_{0}
$$

for all $\lambda \in \mathbb{R}$, which means that

$$
l \cap \operatorname{Gr} \psi_{\alpha}=\emptyset \text { for every } \alpha \in\left(0, \alpha_{0}\right) .
$$

Let us now consider the case of $l \cap K_{1}=\emptyset$. Then $\left(\lambda_{0}, \lambda_{0} \tan t\right) \in K_{2}$, and we have $\lambda_{0} \in\{1-\alpha, 1+\alpha\}$ getting

$$
\lambda_{0} \tan t \in\{\sup \{a:\|(1-\alpha, a)\|=\alpha\}, \sup \{a:\|(1+\alpha, a)\|=\alpha\}\} .
$$

The first one of the above two statements is obvious. Suppose that the latter does not hold. In such a case we obtain a contradiction with condition (2.12). Indeed, the line $l$ contains a point $\left(a_{1}, b_{1}\right)$ such that $a_{1} \in(1-\alpha, 1+\alpha)$ and $b_{1}<f\left(a_{1}\right)$. It is a consequence of the continuity of the function $g: \mathbb{R} \rightarrow \mathbb{R}$ such that $\operatorname{Gr} g=l$ and of Remark 2.15. We have $\left\|\left(a_{1}, b_{1}\right)\right\|<1$, which means that the point $\left(a_{1}, b_{1}\right)$ is an element of $\operatorname{Int} K\left((1,0), \alpha_{0}\right)$.

The converse implication may be proved similarly. 
Now we are able to prove the main result of the present paper. Namely, our next theorem states that if in two normed spaces the generalizations of sinus are the same, then these spaces are essentially the same.

Theorem 2.17. Let $\left(X_{1},\|\cdot\|_{1}\right),\left(X_{2},\|\cdot\|_{2}\right)$ be two normed real linear spaces. Further, let $x_{1}, y_{1} \in X_{1}$ and $x_{2}, y_{2} \in X_{2}$ be such that $\left\|x_{1}\right\|_{1}=\left\|y_{1}\right\|_{1}=$ $\left\|x_{2}\right\|_{2}=\left\|y_{2}\right\|_{2}=1$ and $x_{1} \perp_{B J} y_{1} x_{2} \perp_{B J} y_{2}$. Then the equality

$$
\phi_{x_{1}, y_{1}}^{1}=\phi_{x_{2}, y_{2}}^{2}
$$

implies that

$$
\left\|a x_{1}+b y_{1}\right\|_{1}=\left\|a x_{2}+b y_{2}\right\|_{2}
$$

for all real numbers $a, b$.

Proof. Take unit vectors $x_{1}, x_{2}, y_{1}, y_{2} \in X$ which are respectively Birkhoff-James orthogonal. Denote, for brevity, $\left\|a x_{1}+b y_{1}\right\|_{1}$ by $\|(a, b)\|_{1}$ and $\left\|a x_{2}+b y_{2}\right\|_{2}$ by $\|(a, b)\|_{2}$. Suppose that there exist $a_{0}, b_{0} \in \mathbb{R}$ such that

$$
\left\|\left(a_{0}, b_{0}\right)\right\|_{1} \neq\left\|\left(a_{0}, b_{0}\right)\right\|_{2} .
$$

We are going to show that there exists a $\theta \in[0,2 \pi]$, such that

$$
\phi_{x_{1}, y_{1}}^{1}(\theta) \neq \phi_{x_{2}, y_{2}}^{2}(\theta) .
$$

Note that there is no loss of generality in assuming that $b_{0}>0$;in fact, if $\left\|\left(a_{0}, b_{0}\right)\right\|_{1} \neq\left\|\left(a_{0}, b_{0}\right)\right\|_{2}$, then also $\left\|\left(-a_{0},-b_{0}\right)\right\|_{1} \neq\left\|\left(-a_{0},-b_{0}\right)\right\|_{2}$. Define the sets

$$
S_{1}:=\left\{(a, b): b>0,|a|<1,\|(a, b)\|_{1}=1\right\}
$$

and

$$
S_{2}:=\left\{(a, b): b>0,|a|<1,\|(a, b)\|_{2}=1\right\}
$$

From Lemma 2.13 we know that these sets yield graphs of some concave functions, $f_{0}, g_{0}:(-1,1) \rightarrow \mathbb{R}$, respectively. The first step of the proof is to show that condition (2.13) implies that $S_{1} \neq S_{2}$ and, in consequence, there exists a $t \in(-1,1)$ such that $f_{0}(t) \neq g_{0}(t)$. To this end let us note that

$$
1=\left\|\left(\frac{a_{0}}{\left\|\left(a_{0}, b_{0}\right)\right\|_{1}}, \frac{b_{0}}{\left\|\left(a_{0}, b_{0}\right)\right\|_{1}}\right)\right\|_{1} \neq\left\|\left(\frac{a_{0}}{\left\|\left(a_{0}, b_{0}\right)\right\|_{1}}, \frac{b_{0}}{\left\|\left(a_{0}, b_{0}\right)\right\|_{1}}\right)\right\|_{2} .
$$

If now $\left|\frac{a_{0}}{\left\|\left(a_{0}, b_{0}\right)\right\|_{1}}\right|<1$, then obviously $S_{1} \neq S_{2}$. Note that the case $\left|\frac{a_{0}}{\left\|\left(a_{0}, b_{0}\right)\right\|_{1}}\right|>1$ is impossible since, otherwise, using Remark 2.14, we would obtain

$$
\left\|\left(\frac{a_{0}}{\left\|\left(a_{0}, b_{0}\right)\right\|_{1}}, \frac{b_{0}}{\left\|\left(a_{0}, b_{0}\right)\right\|_{1}}\right)\right\|_{1}>1
$$


which contradicts condition (2.14). Assume that $\left|\frac{a_{0}}{\left\|\left(a_{0}, b_{0}\right)\right\|_{1}}\right|=1$. In this case from (2.14), using the Birkhoff-James orthogonality of $x_{2}$ and $y_{2}$, we obtain

$$
\alpha:=\left\|\left(\frac{a_{0}}{\left\|\left(a_{0}, b_{0}\right)\right\|_{1}}, \frac{b_{0}}{\left\|\left(a_{0}, b_{0}\right)\right\|_{1}}\right)\right\|_{2}>1 \text {. }
$$

Consider the vector

$$
z:=\frac{1}{\alpha}\left(\frac{a_{0}}{\left\|\left(a_{0}, b_{0}\right)\right\|_{1}}, \frac{b_{0}}{\left\|\left(a_{0}, b_{0}\right)\right\|_{1}}\right)=\left(z_{1}, z_{2}\right) .
$$

Thus we have found $z_{1}, z_{2} \in \mathbb{R}$ such that $\left|z_{1}\right|<1,\|z\|_{2}=1$ and $z_{2}>0$. That means $z \in S_{2}$ or, equivalently, $g_{0}\left(z_{1}\right)=z_{2}$. On the other hand $\|z\|_{1}<1$ i.e. $z \notin S_{1}$ and, finally, $f_{0}\left(z_{1}\right) \neq z_{2}$ thus $f_{0}\left(z_{1}\right) \neq g_{0}\left(z_{1}\right)$ for some $z_{1}<1$. Let $f$ and $g$ stand for the continuous extensions of $f_{0}$ and $g_{0}$, respectively, onto the closed interval $[-1,1]$. From Lemma 2.12 we get the existence of a line $b=c a+d_{0}, c \neq 0$ such that this line has a common point with the graph of $f$ and lies above the graph of $g$. We can assume that this function is a supporting line for $f$ (it is enough to replace the original number $d_{0}$ by another one). Denote the abscissa of the contact point by $t_{0}$. Then

$$
f\left(t_{0}\right)=c t_{0}+d_{0} \text { and } c t+d_{0}>g(t)
$$

for all $t \in[-1,1]$. Now take a $d_{1}$ such that the line $b=c a+d_{1}$ is a supporting line for $g$ at a point $t_{1}$; note that $d_{1}<d_{0}$.

Assume that $c>0$; then we have $d_{0}>c$. Indeed, $g(-1) \geq 0$ and

$$
d_{1}+c t \geq g(t)
$$

for all $t \in[a, b]$. In particular $d_{0}-c>d_{1}-c \geq 0$. In the case where $c<0$ one can similarly show that $d_{0}>-c$.

Take an $\alpha \in(0,1]$ and consider the function $\psi_{\alpha}:(1-\alpha, 1+\alpha) \rightarrow \mathbb{R}$ defined by the formula

$$
\psi_{\alpha}(a):=\alpha f\left(\frac{1}{\alpha}(a-1)\right)
$$

(if $c<0$ then we have to consider the similar function with $(a-1)$ replaced by $(a+1)$ and the remaining part of the proof is unchanged). We have

$$
\psi_{\alpha}\left(1+\alpha t_{0}\right)=\alpha f\left(t_{0}\right)
$$

Let us also note that the function $\psi_{\alpha}$ has a supporting line at the point $1+\alpha t_{0}$ with the slope equal to $c$. If $\psi_{\alpha}$ were differentiable at this point we would have

$$
\psi_{\alpha}^{\prime}(a)=\left[\alpha f\left(\frac{1}{\alpha}(a-1)\right)\right]^{\prime}=\alpha \frac{1}{\alpha} f^{\prime}\left(\frac{1}{\alpha}(a-1)\right)=f^{\prime}\left(\frac{1}{\alpha}(a-1)\right),
$$

whence

$$
\psi_{\alpha}^{\prime}\left(1+\alpha t_{0}\right)=f^{\prime}\left(\frac{1}{\alpha}\left(1+\alpha t_{0}-1\right)\right)=f^{\prime}\left(\frac{1}{\alpha} \alpha t_{0}\right)=f^{\prime}\left(t_{0}\right) .
$$


If $\psi_{\alpha}$ were not differentiable at $1+\alpha t_{0}$, then the similar evaluations could be made for the one-sided derivatives and the line with the slope equal to $c$ would be a supporting line for function $\psi_{\alpha}$ if some line with the same slope would be a supporting line for the function $f$ at the point $t_{0}$.

Summarizing, we have two lines $l:=\left\{\left(a, c a+d_{0}\right): a \in \mathbb{R}\right\}$ and $l_{1}:=\left\{\left(a, c a+d_{1}\right): a \in \mathbb{R}\right\}, d_{0} \neq d_{1}$ which are supporting lines for $f$ and $g$, respectively, with the corresponding points of contact: $\left(t_{0}, f\left(t_{0}\right)\right)$ and $\left(t_{1}, g\left(t_{1}\right)\right)$ for some $t_{0}, t_{1} \in[-1,1]$, i.e.

$$
c t_{0}+d_{0}=f\left(t_{0}\right) \quad \text { and } \quad c t_{1}+d_{1}=g\left(t_{1}\right) .
$$

Since $d_{0} \neq 0$ we are able to define $\alpha_{1}:=\frac{c}{d_{0}} ;$ then

$$
\begin{gathered}
\psi_{\alpha_{1}}\left(1+\alpha_{1} t_{0}\right)=\alpha_{1} f\left(\frac{1}{\alpha_{1}}\left(1+\alpha_{1} t_{0}-1\right)\right)=\alpha_{1} f\left(t_{0}\right) \\
=\frac{c}{d_{0}} f\left(t_{0}\right)=\frac{c}{d_{0}}\left(c t_{0}+d_{0}\right)=\frac{c^{2} t_{0}}{d_{0}}+c .
\end{gathered}
$$

On the other hand the line $l^{*}:=\{(a, c a): a \in \mathbb{R}\}$ contains the point $(1+$ $\left.\alpha_{1} t_{0}, c\left(1+\alpha_{1} t_{0}\right)\right)$. We have

$$
c\left(1+\alpha_{1} t_{0}\right)=c+c \alpha_{1} t_{0}=c+\frac{c^{2} t_{0}}{d_{0}}=\psi_{\alpha_{1}}\left(1+\alpha_{1} t_{0}\right),
$$

which means that the line $l^{*}$ contains the point $\left(1+\alpha_{1} t_{0}, \psi_{\alpha_{1}}\left(1+\alpha_{1} t_{0}\right)\right)$. Moreover the slope of this line is equal to $c$, which means that it is a supporting line for $\psi_{\alpha_{1}}$. In view of Lemma 2.16, that means that $\phi_{x_{1}, y_{1}}^{1}(\arctan c)=\alpha_{1}$. In the same way one can prove that $\phi_{x_{2}, y_{2}}^{2}(\arctan c)=\alpha_{2}$ for $\alpha_{2}=\frac{c}{d_{1}}$. Since $d_{0} \neq d_{1}$, functions $\phi_{x_{1}, y_{1}}^{1}$ and $\phi_{x_{2}, y_{2}}^{2}$ do not coincide.

Corrolary 2.18. Let $(X,\|\cdot\|)$ be a real normed linear space. If $x, y \in X$, with $\|x\|=\|y\|=1$ and $x \perp_{B J} y$ are such vectors that $\phi_{x, y}(t)=\sin t$ for all $t \in \mathbb{R}$, then the subspace $\operatorname{lin}(x, y)$ of $X$ is an inner product space.

Corrolary 2.19. Let $(X,\|\cdot\|)$ be a real normed linear space. Then the norm $\|\cdot\|$ comes from an inner product if and only if for all $x, y \in X, x \perp_{B J} y$ with norm equal to 1 , and for every $t \in \mathbb{R}$ one has $\phi_{x, y}(t)=\sin t$.

Corrolary 2.20. Let $(X,\|\cdot\|)$ be a 2-dimensional real normed linear space. Then if for some $x, y \in X$, with $\|x\|=\|y\|=1$ and $x \perp_{B J} y$ we have $\phi_{x, y}=\sin$, then the same equality is true for all vectors $x, y$ satisfying the above properties.

THEOREM 2.21. Let $(X,\|\cdot\|)$ be a real linear normed space. If for every $x, y \in X$, with $\|x\|=\|y\|=1$ such that $x \perp_{B J} y$ and for every $t \in\left(0, \frac{\pi}{2}\right)$ we have

$$
\phi_{x, y}\left(\frac{\pi}{2}+t\right)=\phi_{x, y}\left(\frac{\pi}{2}-t\right)
$$


then $\|\cdot\|$ comes from an inner product.

Proof. Assume that the norm $\|\cdot\|$ does not come from an inner product. Then there exist vectors $x, y \in X$ such that $x \perp_{B J} y$ and $x \not \perp_{J} y$ which additionally satisfy $\|x\|=\|y\|=1$ (see [1]). Since $x$ and $y$ are not James orthogonal, we have

$$
\|y+x\| \neq\|y-x\|
$$

Assume that $\|y+x\|>\|y-x\|$. Similarly as before, for brevity, we shall write $\|(a, b)\|$ instead of $\|a x+b y\|$. Let $f_{0}:(-1,1) \rightarrow \mathbb{R}$ be such a function that

$$
\operatorname{Gr} f_{0}=\left\{(a, b) \in \mathbb{R}^{2}:\|(a, b)\|=1,|a|<1, b>0\right\} .
$$

Extend this function to a continuous function $f:[-1,1] \rightarrow \mathbb{R}$. Note that from $x \perp_{B J} y$ we infer that $\|y-x\| \geq 1$. Thus $\|y+x\|>1$. Now, define $t:=\frac{1}{\|y+x\|} \in(0,1)$; then $\|t x+t y\|=1$ which means that $t=f(t)$. On the other hand

$$
\|t y-t x\|=\left\|\frac{1}{\|x+y\|}(y-x)\right\|=\frac{\|y-x\|}{\|x+y\|}<1,
$$

i.e. $\|(-t, t)\|<1$ and, consequently,

$$
f(-t)>t=f(t)
$$

Since the point $(t, t)$, is an element of the graph of $f$, we can find a supporting line for function $f$ which contains this point. Denote this line by $l:=\{(a, b)$ : $\left.b=c a+d_{0}\right\}$. Then the line $l^{*}:=\left\{(a, b): b=-c a+d_{0}\right\}$ is not a supporting line for $f$ because this line contains the point $(-t, t)$ which lies below the graph of $f$. Now consider the line $l_{1}:=\left\{(a, b): b=-c a+d_{1}\right\}$, where $d_{1}$ is the maximal number $d$ such that the line $b=-c a+d$ contains some points of the graph of $f$, note that $d_{1}>d_{0}$. Consequently, we have

$$
f(t)=c t+d_{0} \text { and } f\left(t_{1}\right)=-c t_{1}+d_{1},
$$

for some $t_{1} \in[-1,1]$. Assume that $c<0$ ( for the positive $c$ the proof runs similarly; the case of $c=0$ is not possible: in such a case $l^{*}=l$ but we know that $l$ is a supporting line for $f$, and $l^{*}$ is not a supporting line for $f)$. Summarizing, the line $l$ contains the point $(t, t) \in \operatorname{Gr} f$, and the line $l_{1}$ contains a point $\left(t_{1}, f\left(t_{1}\right)\right)$ for some $t_{1} \in[-1,1]$. Given an $\alpha>0$ consider the functions

and

$$
\psi_{\alpha}^{+}(x):=\alpha f\left(\frac{1}{\alpha}(x-1)\right), \quad x \in(1-\alpha, 1+\alpha)
$$

$$
\psi_{\alpha}^{-}(x):=\alpha f\left(\frac{1}{\alpha}(x+1)\right), \quad x \in(-1-\alpha,-1+\alpha) .
$$

A line with the slope equal to $-c$ is a supporting line for $\psi_{\alpha}^{+}$in the point $\left(1+\alpha t_{1}, \psi_{\alpha}^{+}\left(1+\alpha t_{1}\right)\right)$ provided that such a line contains this point (the proof is similar to the corresponding part of the proof of Theorem 2.17). Moreover, 
a line with the slope equal to $c$ containing the point $\left(-1+\alpha t, \psi_{\alpha}^{-}(-1+\alpha t)\right)$ is a supporting line for function $\psi_{\alpha}^{-}$at this point. Let us now consider the lines

$$
l^{+}:=\{(a,-c a): a \in \mathbb{R}\} \text { and } l^{-}:=\{(a, c a): a \in \mathbb{R}\}
$$

and define numbers $\alpha^{+}:=-\frac{c}{d_{1}}$ and $\alpha^{-}:=-\frac{c}{d_{0}}$. Note that $\alpha^{+}, \alpha^{-} \in(0,1]$ and $\alpha^{-} \neq \alpha^{+}$, and these numbers are well defined since $d_{0}, d_{1} \neq 0$. Indeed, $d_{1}$ is positive and $c$ is negative thus the considered fraction is positive. On the other hand, since the line $l^{1}$ supports $f$, every point of this line has a norm greater than or equal to 1 . Consequently, we have $\left\|\left(\frac{d_{1}}{c}, 0\right)\right\| \geq 1$. But $\left\|\left(\frac{d_{1}}{c}, 0\right)\right\|=\left|\frac{d_{1}}{c}\right|$ i.e. $\left|\frac{d_{1}}{c}\right| \geq 1$. For the number $\alpha^{-}$we have a similar situation. Write

$$
\begin{gathered}
\psi_{\alpha^{+}}^{+}\left(1+\alpha^{+} t_{1}\right)=\alpha^{+} f\left(\frac{1}{\alpha^{+}}\left(1+\alpha^{+} t_{1}-1\right)\right)=\alpha^{+} f\left(t_{1}\right) \\
=-\frac{c}{d_{1}}\left(-c t_{1}+d_{1}\right)=-c\left(1-\frac{c t_{1}}{d_{1}}\right)=-c\left(1+\alpha^{+} t_{1}\right) .
\end{gathered}
$$

Hence the line $l^{+}$contains the point $\left(1+\alpha^{+} t_{1}, \psi_{\alpha^{+}}^{+}\left(1+\alpha^{+} t_{1}\right)\right)$. Since its slope is equal to $-c$, this line supports $\psi_{\alpha^{+}}^{+}$. In view of Lemma 2.16, it means that

Similarly

$$
\phi_{x, y}(\arctan (-c))=\alpha^{+}
$$

$$
\phi_{x, y}(\pi-\arctan (-c))=\alpha^{-} .
$$

But we have already observed that $\alpha^{+} \neq \alpha^{-}$, which contradicts (2.15) (with $\left.t=\frac{\pi}{2}-\arctan (-c)\right)$.

It is a known fact that if in a normed space every pair $x, y$ of BirkhoffJames orthogonal vectors is Pythagoras orthogonal then this space must be an inner product space (see [1]). Using our results we shall prove this fact under some additional assumption. It is by no means exciting. But we want to mention it because this example shows that using the properties of the function $\phi_{x, y}$ considered in this paper, one can prove facts concerning the properties of normed spaces, which do not involve the function $\phi_{x, y}$ itself.

Corrolary 2.22. Let $(X,\|\cdot\|)$ be a real normed linear space such that for all $x, y \in X$ with $\|x\|=\|y\|$ we have

$$
\min \|x+\lambda(x+y)\|=\min \|y+\lambda(x+y)\| \text {. }
$$

If every two vectors in $X$ which are Birkhoff-James orthogonal are also Pythagoras orthogonal, then the norm $\|\cdot\|$ comes from an inner product.

To prove this corollary it is enough to repeat the proof of Remark 2.9 to get

$$
\phi_{x, y}\left(\frac{\pi}{2}+t\right)=\phi_{x, y}\left(\frac{\pi}{2}-t\right)
$$


for all $x, y \in X$ with $\|x\|=\|y\|, x \perp_{B J} y$ and for every $t \in\left(0, \frac{\pi}{2}\right)$ and then apply Theorem 2.21 .

\section{REFERENCES}

[1] D. Amir, Characterizations of Inner Product Spaces, Birkhäuser Verlag, Basel-BostonStuttgart 1986.

[2] J. Rätz, On orthogonally additive mappings, Aequationes Math. 28 (1985), 35-49.

[3] Gy. Szabó, A conditional Cauchy equation on normed spaces, Publ. Math. Debrecen 42 (1993), 265-271.

[4] Gy. Szabó, Isosceles orthogonally additive mappings and inner product spaces, Publ. Math. Debrecen 46 (1995), 373-384.

[5] T. Szostok, Modified version of Jensen equation and orthogonal additivity, Publ. Math. Debrecen 58 (2001), 491-504.

[6] T. Szostok, On a generalized orthogonal additivity, Bull. Polish Acad. Sci. Math. 49 (2001), 395-408.

T. Szostok

Uniwersytet Śląski

Instytut Matematyki

Bankowa 14

40-007 Katowice, Poland

E-mail: szostok@ux2.math.us.edu.pl

Received: 1.10.2001. 